\title{
CONSTRUÇÃO E CONFIABILIDADE DAS ESCALAS DE QUALIDADE NA INTERAÇÃO FAMILIAR
}

\section{The construction and reliability of the quality familiar interaction scales}

\author{
Lidia Natalia Dobrianskyj Weber ${ }^{a}$, Paulo Müller Prado ${ }^{\text {b }}$ \\ Ana Paula Viezzer Salvador ${ }^{c}$, Olivia Justen Brandenburg ${ }^{d}$
}

\footnotetext{
a Professora do Departamento de Psicologia e do Programa de Pós-Graduação em Educação da UFPR; mestra e doutora em Psicologia Experimental pela USP. Endereço para correspondência: UFPR - Departamento de Psicologia, Curitiba, PR - Brasil, e-mail:lidia@ufpr.br

b Professor do Departamento de Administração Geral e Aplicada; mestre em Administração pela UFPR e doutor em Administração e Marketing pela EAESP-FGV.Curitiba, PR - Brasil, e-mail: pprado@ufpr.br

c Psicóloga e mestre em Educação pela UFPR, Curitiba, PR - Brasil, e-mail: apviezzer@Yahoo.com.br

' Psicóloga e mestranda do Programa de Pós-Graduação em Análise do Comportamento da UEL, Londrina, PR - Brasil, e-mail: oliviajb@onda.com.br
}

\begin{abstract}
Resumo
Atendendo à necessidade de instrumento brasileiro para avaliar aspectos da interação familiar, as Escalas de Qualidade na Interação Familiar (EQIF) foram desenvolvidas e submetidas a análises estatísticas para teste de confiabilidade. O instrumento, direcionado para a criança/adolescente, responde sobre os comportamentos de seus pais, contém 40 itens agrupados em nove escalas (envolvimento, regras e monitoria, comunicação positiva dos filhos, comunicação negativa, punição corporal, clima conjugal positivo, clima conjugal negativo, modelo parental, sentimento dos filhos). Uma amostra de 2.173 participantes com idade entre 9 e 20 anos respondeu ao instrumento EQIF. As análises foram feitas por meio da combinação do valor do alfa de Cronbach com a análise exploratória de componentes principais, o que possibilitou a redução de itens de cada escala e a alteração da estrutura do instrumento até chegar à versão apresentada neste trabalho. A confiabilidade do instrumento, estimada pelo alfa de Cronbach, apresentou-se adequada para as nove escalas (entre 0,6715 e 0,9235). Foram delimitados pontos de corte para o instrumento, o que permitiu investigar famílias com melhor e pior qualidade de interação familiar, ou seja, famílias em situação de proteção $(23,7 \%$ dos participantes) e famílias em situação de risco (24,1\% dos participantes). O EQIF demonstrou boas propriedades psicométricas e pode ser considerado como uma medida válida de qualidade de interação familiar.
\end{abstract}

Palavras-chave: Confiabilidade; Interação familiar; Práticas educativas parentais. 


\begin{abstract}
Given the need for Brazilian instrument to evaluate aspects of the family interaction, the Family Interaction Quality Scales (EQIF) were developed and subjected to a statistical analysis of reliability. This instrument was designed to collect information from children and adolescents about their parent's behavior, and it contains 40 items grouped in nine subscales (involvement, rules and monitoring, children's positive communication, negative communication, corporal punishment, positive marital climate, negative marital climate, parental models, children's feelings toward parents). Participants were 2173 children and adolescents, aged between 9 and 20 years, who completed the EQIF. The combination of the Cronbach's alpha and a principal components analysis was used. It made possible to reduce the number of items in each scale and to change the structure of the instrument until the current version was agreed upon. The reliability of the instrument, estimated by the Cronbach's alpha, was acceptable for all subscales (ranging from 0.6715 to 0.9235). Instrument cut-points were defined, allowing to create categories of families with better and worse interaction, in other words, families in a protective situation (23.7\%) and families in a risk situation (24.1\%). The EQIF showed good psychometric properties and can be considered a valid measure of the family interaction quality.
\end{abstract}

Keywords: Reliability; Family interaction; Parenting practices.

\section{INTRODUÇÃO}

O comportamento dos pais na interação com seus filhos tem sido muito investigado por meio dos estilos parentais ou das práticas educativas parentais. Do ponto de vista teórico, estilos parentais são a expressão dos objetivos, valores e crenças dos pais e diferentes estilos parentais criam diferentes climas emocionais no lar. O estilo parental corresponde a um padrão mais global de comportamento, ao passo que as práticas educativas parentais referem-se a comportamentos definidos por conteúdos específicos e estratégias usadas para suprimir comportamentos considerados inadequados ou para incentivar a ocorrência de comportamentos adequados (Alvarenga, 2001). São inúmeras as estratégias utilizadas pelos pais que são estudadas por pesquisadores de forma isolada com o objetivo de verificar que efeitos acarretam para o desenvolvimento dos filhos. O envolvimento, a apresentação de regras, a monitoria, a comunicação, a punição são exemplos dessas estratégias. Essas práticas parentais e, portanto, estilos parentais, são definidos pelos pais muito cedo na vida da criança; estudos longitudinais revelam que tendem a permanecer estáveis ao longo dos anos e predizer o desenvolvimento dos filhos (Patterson \& Fischer, 2002).
O envolvimento corresponde à participação dos pais na vida dos filhos - o quanto eles os apóiam, são sensíveis às suas reações, estão presentes, disponíveis e demonstram afeto. Dados empíricos mostram que o envolvimento dos pais relaciona-se com aspectos positivos no desenvolvimento dos filhos: prevenção ao uso de álcool, menor nível de agressão, menor risco de problemas de comportamento, boa saúde física, melhor desenvolvimento social, melhor performance em atividades esportivas, desempenho escolar satisfatório, facilidade para percepção de expressão emotiva (Williams, Radin \& Coggins, 1996; Hoyle \& Leff, 1997; Kochanska, 1997; Aviezer, Resnick, Sagi \& Gini, 2002; Montague \& Walker-Andrews, 2002; Sartor \& Youniss, 2002).

Outra prática educativa parental é a apresentação de regras que definem o que a criança deve ou não deve fazer, de que forma e em que momento fazer. A monitoria, por consistir no acompanhamento dos pais em relação ao cumprimento das regras, tem seu estudo geralmente atrelado àqueles sobre apresentação de regras. Davies, McMahon, Flessati e Tiedemann (1984) comprovaram em sua pesquisa que a obediência dos filhos é muito maior se a explicação da regra for clara, incluindo o que e quando se deve fazer e as conseqüências caso a criança obedeça ou desobedeça. Pesquisas 
confirmam o quanto a monitoria dos pais influencia positivamente no desenvolvimento dos filhos, reduzindo principalmente a probabilidade de comportamentos anti-sociais (Patterson, Reid \& Dishion, 1992; Dishion \& Mcmahon, 1998; Cavell, 2000; Diclemente et al., 2001; Pettit, Laird, Dodge, Bates \& Criss, 2001; Reid, Patterson \& Snyder, 2002; Webb, Bray, Getz \& Adams, 2002; Ceballo, Ramirez, Hearn \& Maltese, 2003).

A comunicação entre pais e filhos indica como se caracteriza o diálogo na interação. Por um lado, a comunicação positiva inclui expressão de opiniões, consideração das preferências dos filhos, incentivo a falar sobre problemas, disponibilidade para ouvir os filhos com atenção e empatia. Pesquisas sobre dependências químicas demonstram que a comunicação positiva apresenta-se como um fator protetivo para envolvimento com álcool e drogas (Kaplow, Curran \& Dodge, 2002; Kelly, Comello \& Hunn, 2002), sendo que a presença do diálogo também é facilitadora no tratamento do uso de substâncias químicas (Kafka \& London, 1991).

Por outro lado, a comunicação negativa pode incluir tanto a ausência de diálogo quanto a presença de uma comunicação aversiva. A ausência de diálogo caracteriza-se pela falta de uma comunicação positiva descrita anteriormente; enquanto a presença de comunicação aversiva pode se evidenciar tanto no conteúdo como na forma de expressão, como, por exemplo, ameaças, xingamentos, gritos e humilhações.

O controle psicológico corresponde a um tipo de comunicação negativa. Trata-se de uma manipulação parental intrusiva, que inclui distanciamento, privação de afeto e indução de culpa (Barber, Olsen \& Shagle, 1994). Pesquisas têm demonstrado que este tipo de comportamento parental está relacionado a problemas comportamentais de internalização e de externalização das crianças (Aunola \& Nurmi, 2005, Barber et al., 1994, Casas et al., 2006).

A prática parental de conseqüenciar comportamentos inadequados, com o objetivo de diminuir sua probabilidade de ocorrência, é definida como punição. Dentre as várias formas utilizadas pelos pais para punir (punição corporal, por custo de resposta, time out, retirada de privilégio), a punição corporal apresenta maiores índices de prejuízos no desenvolvimento da criança e do adolescente. Estudos atuais têm comprovado pontos negativos desse tipo de punição na interação com os filhos.
Foram encontradas associações do uso freqüente de punições corporais com comportamentos agressivos dessas crianças com colegas (Strassberg, Dodge, Pettit \& Bates, 1994), depressão, ansiedade, uso de álcool e comportamento anti-social (Turner \& Finkelhor, 1996; Frias-Armenta, 2002).

A educação dos filhos não ocorre apenas pelo uso de estratégias específicas como as práticas parentais. Outros aspectos da interação familiar contribuem para o aprendizado de novos comportamentos das crianças. Um desses aspectos é o modelo parental, que se refere aos comportamentos dos pais que servem de exemplo para os filhos, especialmente aqueles que indicam valores e modelos morais. Os pais ensinam muito para seus filhos por meio de seus próprios comportamentos, pois parte do aprendizado de um indivíduo se dá mediante a modelação (Catania, 1999). Pesquisas confirmam a transmissão intergeracional de comportamentos como a delinqüência (Gorman-Smith, Tolan, Loeber \& Henry, 1998), o abuso de drogas (Kaplow et al., 2002) e o uso de punição corporal (Mosby, Rawls, Meehan, Mays \& Pettinari, 1999; Murphy-Cowan \& Stringer, 1999).

Outro aspecto da interação familiar que interfere no desenvolvimento dos filhos é o clima conjugal, pelo fato de a relação entre pai e mãe influenciar na forma como estes interagem com seus filhos. Quando há dificuldade no relacionamento conjugal, normalmente os pais apresentam comportamentos parentais pouco efetivos (Erel \& Burman, 1995). Há comprovações empíricas de que conflitos conjugais afetam o desenvolvimento dos filhos (Mchale, Rao \& Krasnow, 2000; Bell, Bell \& Nakata, 2001), até mesmo contribuindo para desenvolvimento de comportamentos anti-sociais (Fincham, 1994). Quando há um relacionamento conjugal satisfatório, os pais se mostram mais sensíveis, responsivos e afetivos com seus filhos (Grych, 2002).

As pesquisas apresentadas até o momento mostram que diferentes aspectos de interação familiar podem ser claramente classificados como fatores de risco e proteção, de acordo com o tipo de influência no desenvolvimento do filho. Por definição, os fatores de proteção referem-se às condições ou variáveis que modificam e (ou) melhoram a resposta do indivíduo, diminuindo sua probabilidade de apresentar problemas de desenvolvimento e, ainda, podem proteger o 
indivíduo de eventos ambientais estressores; já os fatores de risco, ao contrário, são condições que aumentam a probabilidade de ocorrência de resultados negativos ou indesejáveis (Reppold, Pacheco, Bardagi \& Hutz, 2002).

Uma revisão de literatura sobre fatores de risco e proteção no desenvolvimento dos filhos apontou o envolvimento e monitoria como sendo os fatores de proteção mais estudados, e a punição física, conflito conjugal e controle psicológico como fatores de risco mais pesquisados (Weber, Tucunduva \& Faria, 2007). O fato de essas práticas educativas serem amplamente estudadas demonstra sua relevância e a necessidade de desenvolver instrumentos que facilitem a investigação sobre este tema.

Sobre a investigação da interação entre pais e filhos, normalmente, questionários e escalas são utilizados para acessar e avaliar quais são os comportamentos dos pais na relação com suas crianças. Há vantagens nesse método: a aplicação é rápida, a disponibilidade de sujeitos para participar é alta e é uma medida passível de padronização (Buri, 1991). Tal padronização é obtida por meio de testes estatísticos que avaliam a confiabilidade do instrumento.

A confiabilidade de um instrumento, sua consistência, é geralmente acessada pelo coeficiente do alfa de Cronbach, o qual leva em conta a inter-relação entre todos os itens de uma escala, verificando se eles estão medindo o mesmo construto (Loewenthal, 2001). Além disso, de acordo com este mesmo autor, a análise exploratória de componentes principais pode ser utilizada com os itens de um instrumento para avaliar a presença de uma ou mais subescalas.

Utilizando tais recursos estatísticos para avaliar as propriedades psicométricas de um instrumento, o presente trabalho tem como objetivo apresentar o processo de elaboração das Escalas de Qualidade na Interação Familiar (EQIF) e os dados de confiabilidade deste instrumento.

\section{MÉTODO}

\section{Participantes}

Participaram da pesquisa 2.173 crianças e adolescentes, sendo que 1.723 eram de escolas públicas e particulares da região de Curitiba (PR); 124 de uma escola particular de Paranaguá (PR);
111 de uma escola particular de Porto União (SC); 215 adolescentes de famílias de baixa renda de Curitiba, beneficiados por duas organizações não-governamentais. A idade variou de 9 a 20 anos (média de 14,19 anos e desvio-padrão de $2,20)$ e, quanto ao gênero, $53 \%$ eram do sexo feminino e $47 \%$ do masculino.

\section{Instrumentos}

Escalas de Qualidade na Interação Familiar (EQIF) consistem em um instrumento elaborado pelos pesquisadores para avaliar algumas práticas educativas parentais e outros aspectos de interação familiar (Anexo A). A versão final contém 40 questões divididas em nove escalas: envolvimento (questões 1, 9, 17, 19, 26, 27, 30, $35)$, regras e monitoria $(18,28,36,39)$, comunicação positiva dos filhos $(4,12,22)$, comunicação negativa $(2,5,10,13,32)$, punição corporal $(20,31,37)$, clima conjugal positivo $(6$, $14,23,33,38)$, clima conjugal negativo $(3,11,21$, $29)$, modelo parental $(7,15,24)$ e sentimento dos filhos $(8,16,25,34,40)$. As questões são propostas para a criança/adolescente responder sobre as atitudes de seus pais (pai e mãe separadamente). São avaliadas pelo sistema Likert de cinco pontos (nunca, quase nunca, às vezes, quase sempre e sempre). Cada escala fornece um escore, podendo ser analisada uma a uma ou em conjunto.

\section{Procedimento}

Após a elaboração do instrumento EQIF, realizou-se um pré-teste com seis crianças, e alguns ajustes foram feitos. $\mathrm{O}$ instrumento foi aplicado coletivamente em sala de aula para participantes de diferentes idades e diferentes instituições.

A diversidade de instituições e idade dos participantes visou garantir a presença de subgrupos com perfis diferentes na pesquisa, aumentando a possibilidade do uso do instrumento para variadas amostras.

\section{Análise dos Dados}

As análises foram feitas mediante combinação do valor do alfa de Cronbach com a análise exploratória de componentes principais. Tal combinação possibilitou a redução de itens de cada escala e a alteração da estrutura do instrumento, modificando o número de escalas existentes. 


\section{RESULTADOS E DISCUSSÃO}

\section{Construção do instrumento EQIF}

A elaboração do instrumento EQIF iniciou-se com a seleção dos construtos que seriam avaliados. As práticas educativas parentais descritas na introdução serviram de base para a formulação das escalas que constituem o instrumento apresentado neste artigo. Além das práticas e dos aspectos de interação familiar, criou-se a escala sobre sentimento dos filhos, a título de exploração, para avaliar como eles se sentem em relação aos comportamentos de seus pais.

O próximo passo consistiu na construção de questões para cada construto selecionado. Os autores optaram por frases que fossem respondidas por crianças e adolescentes sobre seus pais. Isso porque quando são os pais os sujeitos das pesquisas, há maior risco de relatarem seus comportamentos conforme a conveniência social (Holden \& Edwards, 1989). As frases foram avaliadas conforme sua clareza e relevância e submetidas à opinião de quatro juízes, profissionais de psicologia, externos à equipe de autores. Após o pré-teste, foram feitos ajustes de linguagem.

Com uma primeira versão pronta, iniciouse o processo de aplicações e análises estatísticas, passando por quatro versões temporárias e uma final. A primeira versão continha 103 questões divididas em 11 pequenas escalas e foi reduzida até se chegar à versão atual de 40 questões divididas em nove escalas. Para a redução de questões, utilizouse a análise exploratória dos componentes principais em conjunto com o valor do alfa de Cronbach.

Dessa forma, os critérios de exclusão dos itens foram os seguintes: 1- na análise dos componentes principais, com rotação varimax, foram eliminadas as questões que tinham cargas fatoriais elevadas em dois ou mais fatores (Pasquali \& Araújo, 1986); 2- na análise dos componentes principais, com rotação varimax, foram eliminadas as questões que apresentaram valor da comunalidade abaixo de 0,50 (Hair, Anderson, Tatham \& Black, 2005); 3- o teste do alfa de Cronbach apontou itens com baixa contribuição, os quais foram eliminados. No entanto, nem todas as questões com valor de comunalidade abaixo de 0,50 foram retiradas, pois seguiram aos dois outros critérios de carga fatorial adequada e boa consistência interna.

O objetivo foi construir escalas unidimensionais, com questões agrupadas num único fator e valor do alfa adequado. As escalas divididas em fatores somente foram consideradas como fator independente (uma nova escala) se este foi capaz de congregar três ou mais questões com cargas importantes (Pasquali \& Araújo, 1986).

Dessa forma, a versão final ficou com 40 questões divididas em nove escalas: envolvimento, regras e monitoria, comunicação positiva dos filhos, clima conjugal positivo, modelo, sentimento dos filhos, clima conjugal negativo, comunicação negativa, punição corporal. Essas nove escalas finais apresentaram um único fator, de acordo com a análise de componentes principais, o que demonstra que as questões de cada escala estão empiricamente medindo o mesmo construto teórico. Todas as escalas contêm pelo menos três questões, pois este é o número mínimo para representar uma dimensão suficientemente ampla para recolher um conjunto de comportamentos significativos em relação a um conceito psicológico (Pasquali \& Araújo, 1986). Além disso, as nove escalas apresentaram valores do alfa de Cronbach e do KMO (medida de adequação da amostra) adequados, que podem ser visualizados na Tabela 1.

TABELA 1 - Valor do Alfa de Cronbach e do KMO de Cada Escala do Instrumento EQIF

\begin{tabular}{lllll}
\hline Escalas & $\begin{array}{l}\text { Alfa de } \\
\text { Cronbach } \\
\text { do pai }\end{array}$ & $\begin{array}{l}\text { KMO } \\
\text { do pai }\end{array}$ & $\begin{array}{l}\text { Alfa de } \\
\text { Cronbach } \\
\text { da mãe }\end{array}$ & $\begin{array}{l}\text { KMO } \\
\text { da mãe }\end{array}$ \\
\hline Envolvimento & 0,9235 & 0,940 & 0,8902 & 0,926 \\
Regras e monitoria & 0,7940 & 0,761 & 0,6715 & 0,704 \\
Comunicação positiva dos filhos & 0,7780 & 0,692 & 0,7615 & 0,685 \\
Comunicação negativa & 0,7113 & 0,784 & 0,7300 & 0,784 \\
Punição corporal & 0,6918 & 0,671 & 0,7122 & 0,667 \\
Clima conjugal positivo & 0,9134 & 0,875 & 0,9118 & 0,875 \\
Clima conjugal negativo & 0,8031 & 0,789 & 0,7976 & 0,783 \\
Modelo & 0,7807 & 0,697 & 0,6987 & 0,666 \\
Sentimento dos filhos & 0,9104 & 0,891 & 0,8521 & 0,863 \\
\hline
\end{tabular}


$\mathrm{Na}$ Tabela 1, pode-se observar que todas as escalas apresentaram valores do alfa de Cronbach e KMO aceitáveis. Os valores do alfa demonstraram que as questões de cada escala possuem boa consistência interna. A maioria das nove escalas do instrumento apresentou alfa maior que 0,700 e valores acima dessa marca são considerados bons (Loewenthal, 2001). Apenas três escalas (punição corporal do pai, regras e monitoria da mãe, modelo da mãe) apresentaram alfa entre 0,650 e 0,700, sendo que tais valores ainda são considerados acima do limite de aceitabilidade (Hair et al., 2005).

Ao analisar os valores do KMO, podese perceber que a maioria das nove escalas apresentou KMO acima de 0,70, valor considerado bom (Hair et al., 2005). No entanto, três escalas (comunicação positiva dos filhos, punição corporal e modelo, tanto dos pais quanto das mães) apresentaram valores entre 0,65 e 0,70 , considerados ainda adequados (Hair et al., 2005). Para esses autores, o valor do KMO tende a diminuir quando o número de variáveis que mede determinado construto é pequeno. Pode-se verificar, portanto, que as três escalas que apresentaram valores do KMO menores são constituídas por apenas três questões, o que poderia justificar seus valores mais baixos, porém ainda dentro da margem de aceitabilidade.

\section{Pontos de corte do instrumento EQIF}

Considera-se importante a delimitação de pontos de corte para o instrumento, pois estes fornecem um parâmetro de comparação com amostras de outras pesquisas e permitem investigar famílias com melhor qualidade de interação familiar, aqui chamadas de protetivas, e aquelas que apresentam fatores de risco.

As nove escalas do EQIF foram classificadas em "positivas" e "negativas". As escalas positivas são envolvimento, regras e monitoria, comunicação positiva dos filhos, modelo parental, sentimento dos filhos e clima conjugal positivo; enquanto as escalas negativas são punição corporal, comunicação negativa e clima conjugal negativo. Tal classificação foi baseada em pesquisas que mostraram que tais práticas podem representar fatores de risco ou proteção para o desenvolvimento dos filhos (Weber et al., 2007).
Os valores de corte foram calculados seguindo-se alguns passos:

a) total Positivo (TP): somaram-se os escores das seis escalas positivas;

b) total Negativo (TN): somaram-se os escores das três escalas negativas;

c) calcularam-se os percentis 40 e 60 do TP e do TN.

Assim, caracterizaram-se como famílias protetivas aquelas que apresentaram escores altos (acima do percentil 60) nas práticas positivas e escores baixos (abaixo do percentil 40) nas negativas; e como famílias de risco aquelas cujos escores foram baixos (abaixo do percentil 40) nas escalas positivas e altos (acima do 60) nas negativas.

Esses cálculos foram realizados de forma independente para crianças (participantes com até 12 anos) e para adolescentes (participantes com idade igual/acima de 13 anos). Dessa forma, definiu-se um ponto de corte para crianças e outro para adolescentes, visto que esses últimos tendem a apresentar escores significativamente diferentes do que os das crianças em todas as escalas do EQIF $(\mathrm{t}=14,056$ para envolvimento; $\mathrm{t}=8,403$ para regras e monitoria; $\mathrm{t}=15,857$ para comunicação positiva dos filhos; $\mathrm{t}=-6,104$ para comunicação negativa; $\mathrm{t}=10,759$ para punição corporal; $\mathrm{t}=8,222$ para clima conjugal positivo; $\mathrm{t}$ $=-2,607$ para clima conjugal negativo; $\mathrm{t}=11,765$ para modelo parental; e $\mathrm{t}=11,388$ para sentimento dos filhos; todos com $\mathrm{p}<0,001)$. Verificou-se que nas escalas de comunicação negativa e clima conjugal negativo, os adolescentes apresentaram médias significativamente mais altas que as crianças; nas outras sete escalas, os adolescentes apresentaram médias significativamente mais baixas que as crianças.

Os pontos de corte encontrados podem ser verificados a seguir:

- Crianças com fatores de proteção: TP igual e maior que 259 e TN igual e menor que 42 .

- Crianças com fatores de risco: TP igual e menor que 245 e TN igual e maior que 49.

- Adolescentes com fatores de proteção: TP igual e maior que 227 e TN igual ou menor que 42.

- Adolescentes com fatores de risco: TP igual e menor que 205 e TN igual e maior que 50. 
A utilização desses pontos de corte deve ser cuidadosa. Os valores apresentados acima devem ser usados preferencialmente como parâmetro de comparações ou como indicativos da qualidade na interação familiar. Utilizá-los como critério de "diagnóstico", único e isolado, pode acarretar erros ou equívocos, uma vez que os valores de corte podem ser diferentes em outras amostras.

Verificou-se, depois da delimitação dos pontos de corte, que $23,7 \%$ das famílias de crianças e adolescentes da presente amostra encontravam-se em situação de proteção e $24,1 \%$ das famílias em situação de risco; as demais famílias $(52,2 \%)$ encontraram-se em uma faixa intermediária. As famílias em situação de proteção apresentaram média significativamente superior que as famílias em situação de risco nas seis escalas positivas ( $\mathrm{p}<0,001)$, e apresentaram média significativamente inferior que as famílias de risco nas três escalas negativas $(p<0,001)$.

\section{CONSIDERAÇÕES FINAIS}

Diante da construção minuciosa do instrumento proposto no presente trabalho, do embasamento teórico bastante denso e dos dados obtidos, pode-se afirmar que o objetivo dos pesquisadores foi atingindo favoravelmente e os resultados alcançados foram satisfatórios.

Certamente há diversos instrumentos que avaliam interação pais-filhos. O instrumento EQIF foi elaborado e testado porque muitas escalas utilizadas no Brasil foram traduzidas de outras línguas e culturas. Outra vantagem do instrumento EQIF é a possibilidade de avaliar maior número de aspectos de interação familiar e de forma mais detalhada. Apesar disso, deve-se atentar para limitações próprias de instrumentos baseados no relato do participante.

Acrescenta-se que o instrumento está sendo submetido a novas aplicações e a análises mais aprofundadas, com o propósito de desenvolver ainda mais as nove escalas. Também serão realizados testes como Análise Fatorial Confirmatória e Equações Estruturais.

Os dados encontrados até o presente momento já demonstram a confiabilidade do instrumento EQIF. Com a confiabilidade verificada, o instrumento pode ser utilizado por outros pesquisadores, como também por profissionais que queiram identificar possíveis fatores de risco e (ou) proteção em famílias a fim de direcionar e facilitar suas orientações e (ou) intervenções familiares.

\section{AGRADECIMENTO}

Pesquisa realizada com o apoio da Fundação Araucária e Conselho Nacional de Desenvolvimento Científico e Tecnológico (CNPq).

Nossa gratidão à Prof. ${ }^{a}$ Dr. ${ }^{a}$ Acácia Aparecida Angeli dos Santos, pela cuidadosa leitura e sugestões essenciais sobre o texto.

\section{REFERÊNCIAS}

Alvarenga, P. (2001). Práticas educativas parentais como forma de prevenção de problemas de comportamento. In H. J. Guilhardi (Org.). Sobre comportamento e cogniçãa. (Vol. 8, pp. 54-60). Santo André: ESETec.

Aunola, K., \& Nurmi, J. E. (2005). The role parenting styles in children's problem behavior. Child Development, 76 (6), 1144-1159.

Aviezer, O., Resnick, G., Sagi, A., \& Gini, M. (2002). School competence in young adolescence: Links to early attachment relationships beyond concurrent selfperceived competence and representations of relationships. International Journal of Behavioral Development, 26(3), 397-409.

Barber, B. K., Olsen, J. E., \& Shagle, S. C. (1994). Associations between parental psychological and behavioral control and youth internalized and externalized behaviors. Child Development, 65, 1120-1136.

Bell, L. G., Bell, D. C., \& Nakata, Y. (2001). Triangulation and Adolescent Development in the U.S. and Japan. Family Process, 40, 173-186.

Buri, J. R. (1991). Parental authority questionnaire. Journal of Personality Assessment, 57, 110-119. 
Casas, J. F., Weigel, S. M., Crick, N. R., Ostrov, J. M., Woods, K. E., Yeh, E. A. J., et al. (2006). Early parenting and children's relational and physical aggression in the preschool and home contexts. Applied Developmental Psychology, 27(3), 209-227.

Catania, C. A. (1999). Aprendizagem. Porto Alegre: Artmed.

Cavell, T. A. (2000). Working with parents of aggressive children: A practitioner's guide. Washington: American Psychological Association.

Ceballo, R., Ramirez, C., Hearn, K. D., \& Maltese, K. L. (2003). Community Violence and Children's Psychological Well-Being: Does Parental Monitoring Matter? Journal of Clinical Child \& Adolescent Psychology, 32(4), 586-592

Davies, G. R., McMahon, R. J., Flessati, E. W., \& Tiedemann, G. L. (1984). Verbal rationales and modeling as adjuncts to a parenting technique for child compliance. Child Development, 55, 1290-1298.

DiClemente, R. J., Wingood, G. M., Crosby, R., Sionean, C., Cobb, B. K., Harrington, K., et al. (2001). Parental monitoring: Association with adolescents' Risk Behaviors pediatrics. Recovered in 10 mar. 2004, from (Word Wide Web): http://www.findarticles.com/cf_dls/ m0950/6_107/75561202/p1/article.jhtml

Dishion, T. J., \& McMahon, R. J. (1998). Parental monitoring and the prevention of child and adolescent problem behavior: A conceptual and empirical formulation. Clinical Child and Family Psychology Review, 1, 61-75.

Erel, O. \& Burman, B. (1995). Interrelatedness of marital relations and parent-child relations: A meta-analytic review. Psychological Bulletin, 118, 108-132.

Fincham, F. D. (1994). Understanding the association between marital conflict and child adjustment: Overview. Journal of Family Psychology, 8, 123-127.

Frias-Armenta, M. (2002). Long-term effects of child punishment on Mexican women: A structural model. Child Abuse and Neglect, 26(4), 371-386.
Gorman-Smith, D., Tolan, P. H., Loeber, R. \& Henry, D. B. (1998). Relation of family problems to patterns of delinquent involvement among urban youth. Journal of Abnormal Child Psychology. Recovered in 09 mar. 2004, from (Word Wide Web): http:/ /www.findarticles.com/cf_dls/m0902/ 5_26/53286529/p1/article.jhtml

Grych, J. H. (2002). Marital relationships and parenting. In M. H. Bornstein (Org.). Hanbook of parenting. (pp. 203-225). New Jersey: Lawrence Erlbaum Associates

Hair, J. F., Anderson, R. E., Tatham, R. E., \& Black, W.C. (2005). Análise Multivariada de dados. Porto Alegre: Bookman.

Holden, G. W., \& Edwards, L. A (1989). Parental attitudes toward child rearing: instruments, issues and implications. Psychological Bulletin, 106, 29-58.

Hoyle, R. H., \& Leff, S. S. (1997). The role of parental involvement in youth sport participation and performance. Adolescence, 32(125), 233-243

Kafka, R. ,\& London, P. (1991). Communication in relationships and adolescent substance use: The influence of parents and friends. Adolescence, 26(103), 587-598.

Kaplow, J. B., Curran, P. J., \& Dodge, K. A. (2002). Child, parent, and peer predictors of early-onset substance use: a multisite longitudinal study. Journal of Abnormal Child Psychology. 3,199-216 . Recovered in 18 mar. 2004, from (Word Wide Web): http:/ / www.findarticles.com/cf_dls / m0902/3_30/86874928/p1/article.jhtml

Kelly, K.J., Comello, M.L.G., \& Hunn, L.C.P. (2002). Parent-child communication, perceived sanctions against drug use, and youth drug involvement. Adolescence. Recovered In 18 mar. 2004, from (Word Wide Web): http://www.findarticles.com/cf_0/ m2248/148_37/97723212/p1/article.jhtml

Kochanska, G. (1997). Mutually responsive orientation between mothers and their young children: implication for early socialization. Child Development, 68, 94-112. 
Loewenthal, K.M. (2001). An introduction to psychological tests and scales. London: Psychology Press.

McHale, J.P., Rao, N., \& Krasnow, A.D. (2000). Constructing family climates: Chinese mothers' reports of their co-parenting behaviour and preschoolers' adaptation. International Journal of Behavioral Development, 24(1), 111-118.

Montague, D.P.F., \& Walker-Andrews, A.S. (2002). Mothers, fathers, and infants: The role of person familiarity and parental involvement in infants' perception of emotion expressions. Child Development, 73(5), 1339-1352.

Mosby, L., Rawls, A.W., Meehan, A.J., Mays, E., \& Pettinari, C.J. (1999). Troubles in interracial talk about discipline: An examination of African American child rearing narratives. Journal of Comparative Family Studies, 30(3), 489-521.

Murphy-Cowan, T., \& Stringer, M. (1999). Physical punishment and the parenting cycle: A survey of Northern Irish parents. Journal of Community and Applied Social Psychology, 9(1), 61-71.

Pasquali, L. \& Araújo, J.M.A. (1986). Questionário de percepção para pais: Q.P.P. Psicologia: Teoria e Pesquisa, 2(1), 56-72.

Patterson, G. R., \& Fischer (2002). Recent developments in our understanding of parenting: bidirectional effects, causal models and the search for parsimony. In M. Bornstein (Org.). Handbook of parenting - Practical issues. (pp. 59-88) Mahwah/New Jersey: Lawrence Erlbaum Associates.

Patterson, G. R., Reid, J. B., \& Dishion, T. J. (1992). A social interactional approach. Vol. 4: Antisocial boys. Eugene: Castalia.

Pettit, G. S., Laird, R. D., Dodge, K. A., Bates, J. E., \& Criss, M. M. (2001). Antecedents and behaviorproblem outcomes of parental monitoring and psychological control in early adolescence. Child Development, 72(2), 583-598.

Reid, J. B., Patterson, G. R., \& Snyder, J. (2002). Antisocial Behavior in Children and Adolescent. Washington: American Psychological Association.
Reppold, C. T., Pacheco, J., Bardagi, M., \& Hutz, C. S. (2002). Prevenção de problemas de comportamento e desenvolvimento de competências psicossociais em crianças e adolescentes: uma análise das práticas educativas e dos estilos parentais. In C. S. Hutz (Org.). Situações de risco e vulnerabilidade na infância e adolescência: Aspectos teóricos e estratégias de intervenção ( $\mathrm{pp}$. 9-51). São Paulo: Casa do Psicólogo.

Richardson, R. J. (1999). Pesquisa social: Métodos e técnicas. São Paulo: Atlas.

Sartor, C. E., \& Youniss, J. (2002). The relationship between positive parental involvement and identity achievement during adolescence. Adolescence, 37(146), 221-234.

Strassberg, Z., Dodge, K. A., Pettit, G. S., \& Bates, J. E. (1994). Spanking in the home and children's subsequent aggression toward kindergarten peers. Development and Psychopathology, 6(3), 445-461.

Turner, H.A., \& Finkelhor, D. (1996). Corporal punishment as a stressor among youth. Journal of Marriage and the Family, 58(1), 155-166.

Webb, J.A., Bray, J.H., Getz, J.G., \& Adams, G. (2002). Gender, perceived parental monitoring and behavioral adjustment: Influences on adolescent alcohol use. American Journal of Orthopsychiatry, 72(3), 392-400.

Weber, L.N.D., Tucunduva, C., \& Faria, R. (2007). Fatores de Risco e Proteção para a Criança na Interação Familiar - Revisão de Literatura. In Resumo, 16. Encontro Brasileiro de Psicoterapia e Medicina Comportamental, 2007, Brasília. Brasília: Associação Brasileira de Psicoterapia e Medicina Comportamental.

Williams, E., Radin, N., \& Coggins, K. (1996). Parental involvement in childrearing and the school performance of Ojibwa children: An exploratory study. Merrill-Palmer Quarterly, 42(4), 578-595.

Recebido: 14/02/2008 Received: 02/14/2008

Aprovado: 03/03/2008 Approved: 03/03/2008 


\section{ANEXO A \\ EQIF - ESCALAS DE QUALIDADE NA INTERAÇÃO FAMILIAR}

Caro(a) participante: A sua ajuda é muito importante para a nossa pesquisa sobre a família, mas lembramos que você só participa se quiser. Pedimos que você responda sinceramente a todas as questões, sem deixar nenhuma em branco. Não existem respostas certas ou erradas! Você não precisa escrever o seu nome e ninguém mais, além das pesquisadoras, saberá as suas respostas. Muito obrigada pela sua colaboração!

Idade:

Sexo: ( ) feminino (

) masculino Com quem você mora?

$\rightarrow$ Responda as seguintes questões sobre o seu pai e sobre a sua mãe (ou sobre as pessoas por quem foi educado, por exemplo: madrasta, padrasto, avô, avó, tio, tia e outros). Numere de 1 a 5 de acordo com a tabela abaixo:
(1) $=$ Nunca
(2) $=$ Quase nunca
(3) $=$ Às vezes
(4) = Quase sempre
(5) $=$ Sempre

1. Meus pais costumam dizer o quanto eu sou importante para eles.

PAI ( ) MÃE ( )

2. Meus pais brigam comigo por qualquer coisa.

PAI ( ) MÃE ( )

3. Meus pais costumam xingar um ao outro.

PAI ( ) MÃE ( )

4. Eu costumo contar as coisas boas que me acontecem para meu pai/minha mãe.

PAI ( ) MÃE ( )

5. Meus pais costumam falar alto ou gritar comigo.

PAI ( ) MÃE ( )

6. Meus pais fazem carinho um no outro.

PAI ( ) MÃE ( )

7. O que meus pais me ensinam de bom eles também fazem.

PAI ( ) MÃE ( )

8. Eu penso que meu pai/minha mãe são os melhores pais que eu conheço.

PAI ( ) MÃE ( )

9. Meus pais ficam felizes quando estão comigo.

PAI ( ) MÃE ( )

10. Meus pais costumam descontar em mim quando estão com problemas.

PAI ( ) MÃE ( )

11. Meus pais falam mal um do outro.

PAI ( ) MÃE ( )

12. Eu costumo contar as coisas ruins que me acontecem para meu pai/minha mãe.

PAI ( ) MÃE ( )

13. Meus pais costumam me xingar ou falar palavrões para mim.

PAI ( ) MÃE ( )

14. Meus pais fazem elogios um para o outro.

PAI ( ) MÃE ( ) 
15. Meus pais também fazem as obrigações que me ensinam.

PAI ( ) MÃE ( )

16. Eu me sinto amado pelos meus pais.

PAI ( ) MÃE ( )

17. Meus pais procuram saber o que aconteceu comigo quando estou triste.

PAI ( ) MÃE ( )

18. Meus pais sabem onde eu estou quando não estou em casa.

PAI ( ) MÃE ( )

19. Quando ajudo meus pais, eles me agradecem.

PAI ( ) MÃE ( )

20. Meus pais costumam me bater quando faço alguma coisa errada.

PAI ( ) MÃE ( )

21. Meus pais costumam estar brabos um com o outro.

PAI ( ) MÃE ( )

22. Eu costumo falar sobre meus sentimentos para meu pai/minha mãe.

PAI ( ) MÃE ( )

23. Meus pais costumam se abraçar.

PAI ( ) MÃE ( )

24. Eu acho legais as coisas que meus pais fazem.

PAI ( ) MÃE ( )

25. Meus pais são um bom exemplo para mim.

PAI ( ) MÃE ( )

26. Meus pais costumam mostrar que se preocupam comigo.

PAI ( ) MÃE ( )

27. Meus pais demonstram orgulho de mim.

PAI ( ) MÃE ( )

28. Meus pais sabem o que eu faço com o meu tempo livre.

PAI ( ) MÃE ( )

29. Meus pais brigam um com o outro.

PAI ( ) MÃE ( )

30. Meus pais costumam me fazer carinhos quando eu me comporto bem.

PAI ( ) MÃE ( )

31. Meus pais costumam me bater sem eu ter feito nada de errado.

PAI ( ) MÃE ( )

32. Meus pais costumam me criticar de forma negativa.

PAI ( ) MÃE ( )

33. Meus pais falam bem um do outro.

PAI ( ) MÃE ( )

34. Sinto orgulho de meus pais.

PAI ( ) MÃE ( )

35. Meus pais costumam me dar beijos, abraços ou outros carinhos.

PAI ( ) MÃE ( )

36. Meus pais costumam me dar conselhos.

PAI ( ) MÃE ( )

37. Meus pais costumam me bater por coisas sem importância.

PAI ( ) MÃE ( )

38. Meus pais têm bom relacionamento entre eles.

PAI ( ) MÃE ( )

39. Meus pais pedem para eu dizer para onde eu estou indo.

PAI ( ) MÃE ( )

40. Qual a nota que você dá para seus pais, de um a cinco:

$\operatorname{PAI}(\quad) \quad \mathrm{MÃE}(\quad)$ 